\title{
Association between diabetic retinopathy in type 2 diabetes and the ICAM- 1 rs5498 polymorphism: a meta-analysis of case- control studies
}

\author{
Zikang Xie and Hao Liang ${ }^{*}$ (1)
}

\begin{abstract}
Background: Genetic studies have reported contradictory results on the association between the intercellular adhesion molecule-1 (ICAM-1) rs5498 polymorphism and diabetic retinopathy (DR) risk in type 2 diabetic patients. We aimed to perform a systematic literature search and conduct random-effects meta-analysis to provide a quantitative evaluation.

Methods: We searched Pubmed, Embase, Scopus, Web of Science and Wanfang databases from inception up to January 2018. Allelic and genotype frequencies of rs5498 was compared between DR cases and controls. Odds ratios (OR) and 95\% confidence intervals (Cl) were calculated using a random effects model.

Results: Nine studies involving a total of 1792 cases and 1400 controls met our inclusion criteria. We did not find any significant association between rs5498 and DR risk at the dominant model (GG + GA versus AA, OR=1.00, 95\% Cl: $0.66-1.50, P=0.987$ ), the recessive model (GG versus $\mathrm{GA}+\mathrm{AA}, \mathrm{OR}=1.24,95 \% \mathrm{Cl}: 0.86-1.77, P=0.245$ ), the $\mathrm{GG}$ versus $\mathrm{AA}$ contrast ( $O R=1.14,95 \% \mathrm{Cl}: 0.68-1.92, P=0.611)$, and the $\mathrm{G}$ allele versus $\mathrm{A}$ allele contrast $(\mathrm{OR}=1.08,95 \% \mathrm{Cl}: 0.81-1.45$, $P=0.592$ ). Subgroup analysis by ethnicity showed no association in Asian populations ( $G$ allele versus $A$ allele: $O R=1$. 05, 95\% Cl: $0.76-1.44, P=0.790)$. Subgroup analysis by DR subtype also did not reveal any association of rs5498 with proliferative DR ( $G$ allele versus A allele: $\mathrm{OR}=1.34,95 \% \mathrm{Cl}: 0.71-2.52, P=0.364$ ) and non-proliferative DR ( $\mathrm{G}$ allele versus A allele: $\mathrm{OR}=0.71,95 \% \mathrm{Cl}: 0.43-1.17, P=0.180)$.
\end{abstract}

Conclusion: Our meta-analyses provide no evidence of the association of rs5498 with DR in type 2 diabetic patients.

Keywords: ICAM-1, rs5498, Meta-analysis, Diabetic retinopathy

\section{Background}

Diabetic retinopathy (DR) is the single most common complication of diabetes mellitus and the leading cause of blindness in working-aged adults worldwide [1]. Despite numerous breakthroughs in the development of novel pharmacological agents for DR in the last decade, the incidence of DR remains high and 90\% of type 1 and $60 \%$ of type 2 diabetes patients suffer from the disease. Body mass index (BMI), increased duration of diabetes, ineffective blood glucose control, and ineffective blood pressure control are the major risk factors for DR $[1,2]$.

\footnotetext{
* Correspondence: liangh@gxmu.edu.cn

Department of Ophthalmology, First Affiliated Hospital, Guangxi Medical University, Nanning, China
}

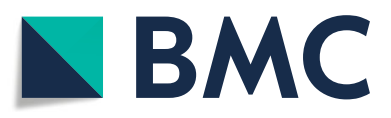

(c) The Author(s). 2018 Open Access This article is distributed under the terms of the Creative Commons Attribution 4.0 International License (http://creativecommons.org/licenses/by/4.0/), which permits unrestricted use, distribution, and reproduction in any medium, provided you give appropriate credit to the original author(s) and the source, provide a link to the Creative Commons license, and indicate if changes were made. The Creative Commons Public Domain Dedication waiver (http://creativecommons.org/publicdomain/zero/1.0/) applies to the data made available in this article, unless otherwise stated.

However, they do not adequately predict disease progression in individual patients, suggesting the presence of a genetic component. Identification of the specific genetic risk factors for DR susceptibility is an area of substantial research and could unravel druggable targets for the purpose of treatment or even prevention.

In recent years, emerging evidence has highlighted the potential role of intercellular adhesion molecule-1 (ICAM-1) in the development of DR. ICAM-1 is a immunoglobulin-(Ig)-like transmembrane glycoprotein expressed on the surface of leukocytes, endothelial cells, and epithelial cells [3]. It influences the adhesion of circulating immune cells to the endothelium and contributes to immune cell migration and perivascular infiltration. Increased levels 
of ICAM-1 and its ligands have been observed in patients with DR and retina of animal models [4-7]. ICAM-1 blockade with monoclonal antibodies effectively prevents diabetic retinal leukostasis, vascular leakage, and capillary nonperfusion in experimental DR [4]. Similarly, when the bioactivity of the ICAM-1 counter receptor CD18 is inhibited, diabetic retinal leukocyte adhesion is potently suppressed [5].

Because ICAM-1 has been implicated in DR development, multiple studies have investigated how genetic variation at ICAM-1 is related to DR risk. More than 100 single-nucleotide polymorphisms (SNPs) were identified in the ICAM-1 gene. The best studied SNP is a G/A polymorphism in exon6 at codon 469 (rs5498), resulting in a lysine (Lys) to glutamine (Glu) substitution in Ig-like domain 5 that is essential for dimerisation, surface presentation and solubilisation of the protein [8]. This polymorphism has been shown to influence the interaction of ICAM-1 with leukocyte function-associated antigen-1 (LFA-1) and the macrophage-1 antigen during leukocyte adhesion [8]. In the present study, we aimed to provide a quantitative evaluation of the association between DR in type 2 diabetes and the ICAM-1 rs5498 polymorphism.

\section{Methods}

\section{Literature search}

The search strategy for this meta-analysis was comprehensive, aiming to retrieve the largest possible number of relevant studies. We systematically screened 5 electronic databases including Pubmed (Additional file 1), Embase, Scopus, Web of Science and Wanfang for articles published between January 1990 and January 2018. The following keywords were used: intercellular adhesion molecule-1, K469E, rs5498, diabetic retinopathy, type 2 diabetes, and polymorphism. In addition, the reference lists of all the retrieved papers and relevant reviews were manually searched for eligible papers. We only included published studies with full-text articles available. In case of overlap between articles reporting on the same cohort, we included the study with the largest cohort. Our meta-analysis adhered to the Preferred Reporting Items for Systematic Reviews and Meta-Analyses (PRISMA) Statement (Additional file 2) [9].

\section{Inclusion and exclusion criteria}

One reviewer performed the initial screen of all papers identified by the electronic searches. Studies were excluded when the title clearly indicated that it did not meet the inclusion criteria. Where a title/abstract could not be rejected with certainty, the full text of the publications was obtained for assessment. Studies were considered eligible if they met the following criteria: 1) evaluated the frequency of the ICAM-1 rs5498 polymorphism in relation to the number of retinopathy cases and controls; 2) published in English or Chinese; and 3) published prior to January 2018 unless an online version of the study had been released prior to this date; and 4) reported odds ratios (ORs) and 95\% confidence intervals (95\% CIs) or data to calculate them. Case-only and case series studies with no control population were excluded, as well as studies based only on phenotypic tests, reviews, meta-analysis. We also excluded unpublished studies or gray literature because we expected them to contain insufficient reporting for our analysis.

\section{Data extraction and quality assessment}

Data extraction was performed by the first author and entered into predesigned electronic tables. The second author checked the extracted data. Disagreements were resolved by discussion between the two authors. The following items were considered: first author, year of publication, location of the study, ethnicity, number of cases and controls, diagnostic criteria, allele or genotype frequency, Hardy-Weinberg equilibrium (HWE) status, and genotyping method. The methodological quality of each study was assessed by the Newcastle-Ottawa Scale, which was used for its simplicity in comparing observational studies. Studies were evaluated based on cohort selection, comparability and ascertainment of exposure using nine multiple-choice questions. Studies were deemed of low quality if the total score was 5 or lower [10].

\section{Statistical analyses}

Statistical analysis was performed using STATA 11 (StataCorp, College Station, TX). To assess HWE status, we used a publicly available program (http://ihg.gsf.de/cgi-b in/hw/hwa1.pl). For our main analysis, we compared allele frequencies (the -encoding allele $G$ versus the -encoding allele $\mathrm{A}$ ) between cases and controls. We also evaluated a dominant model (GG + AG versus AA) and a recessive model (GG versus $A G+A A$ ) for the $G$ allele. All associations were presented as ORs with their corresponding 95\% CIs. Heterogeneity was evaluated by Cochran's $\mathrm{Q}$ and the $\mathrm{I}^{2}$ statistic. When heterogeneity was high $\left(\mathrm{I}^{2}>50 \%, P<0.10\right)$, a pooled analysis was conducted using DerSimonian and Laird random effects models [11]. The significance of the summary OR was determined using an asymptotic Z-test. We evaluated publication bias or selective reporting by using funnel plots as well as Egger's regression intercept test.

\section{Results}

\section{Study characteristics}

Our search yielded 476 records, with 14 articles being possibly eligible after review on abstract level. After full-text review, we excluded 5 studies. Overall, 9 studies involving 1792 cases and 1400 controls met the selection criteria and could be used for meta-analysis [12-20]. 
Figure 1 showed the process of identifying eligible studies. The mean (range) year of publication was 2010 (2002-2016). The mean (range) sample size was 355 (70-792). Studies had been performed in 4 countries, including China $(n=5)$, India $(n=2)$, Japan $(n=1)$, and Slovenia $(n=1)$. Study characteristics and methodological quality of included studies are shown in Table 1.

\section{Data synthesis}

The minor allele frequency (MAF) for the ICAM-1 rs5498 polymorphism varied from 16.7 to $54.8 \%$. The pooled effect estimates among all studies did not find statistically significant associations between the ICAM-1 rs5498 polymorphism and retinopathy in type 2 diabetes at the dominant model $(\mathrm{GG}+\mathrm{GA}$ versus $\mathrm{AA}, \mathrm{OR}=1.00$, 95\% CI: $0.66-1.50, P=0.987$ ), the recessive model (GG versus $\mathrm{GA}+\mathrm{AA}, \mathrm{OR}=1.24,95 \% \mathrm{CI}: 0.86-1.77, P=$ $0.245)$, the $G G$ versus $A A$ contrast $(O R=1.14,95 \% \mathrm{CI}$ : $0.68-1.92, P=0.611)$, and the $G$ allele versus $A$ allele contrast $\quad(\mathrm{OR}=1.08, \quad 95 \% \quad \mathrm{CI}: \quad 0.81-1.45, \quad P=0.592)$ (Table 2 and Figs. 2 and 3). Among the included studies, 8 studies with 1597 cases and 1257 controls were performed on Asian populations. We conducted subgroup analyses by ethnicity using Asian studies, but we did not find any significant associations of the ICAM-1 rs5498 polymorphism with retinopathy in Asians (dominant model: $\mathrm{OR}=0.96,95 \% \mathrm{CI}$ : $0.61-1.50, P=0.843$; recessive model: OR $=1.15$, 95\% CI: $0.79-1.68, P=0.469$; GG versus AA contrast: $\mathrm{OR}=1.04,95 \% \mathrm{CI}: 0.60-1.81, P=$
0.887; $\mathrm{G}$ allele versus A allele: $\mathrm{OR}=1.05,95 \% \mathrm{CI}$ : $0.76-$ 1.44, $P=0.790$ ) (Table 2 and Figs. 2 and 3). The single Caucasian study showed a statistically significant association between rs5498 and retinopathy at the recessive model $(\mathrm{OR}=2.00,95 \% \mathrm{CI}: 1.15-3.48, P=0.014)$, the GG versus $A A$ contrast $(\mathrm{OR}=2.21,95 \% \mathrm{CI}: 1.16-4.22, P=$ $0.016)$ and the $G$ allele versus $A$ allele contrast $(\mathrm{OR}=$ 1.44, 95\% CI: 1.06-1.95, P=0.021) (Table 2) [14]. When DR was subdivided into non-proliferative DR and proliferative DR, subgroup analysis did not show evidence of significant associations (Table 2). The influential analysis for the $I C A M-1$ rs5498 polymorphism revealed that there was no single study which significantly influenced the overall results (Fig. 4).

\section{Heterogeneity and publication bias}

The present meta-analysis revealed heterogeneity among the included studies ( $\mathrm{I}^{2}$ ranged from $65.3-84.0 \%$ ). The funnel plot did not demonstrate apparent asymmetry (Fig. 5). Egger's test also did not indicate any evidence of publication bias (Table 3 ).

\section{Discussion}

DR is the most frequent microvascular complication from type 2 diabetes. A large body of clinical and experimental literature has indicates that leucocyte adhesion to the retinal vasculature plays an important role in the pathogenesis of DR. As a central mediator of leukocyte adhesion to and transmigration across the endothelium,

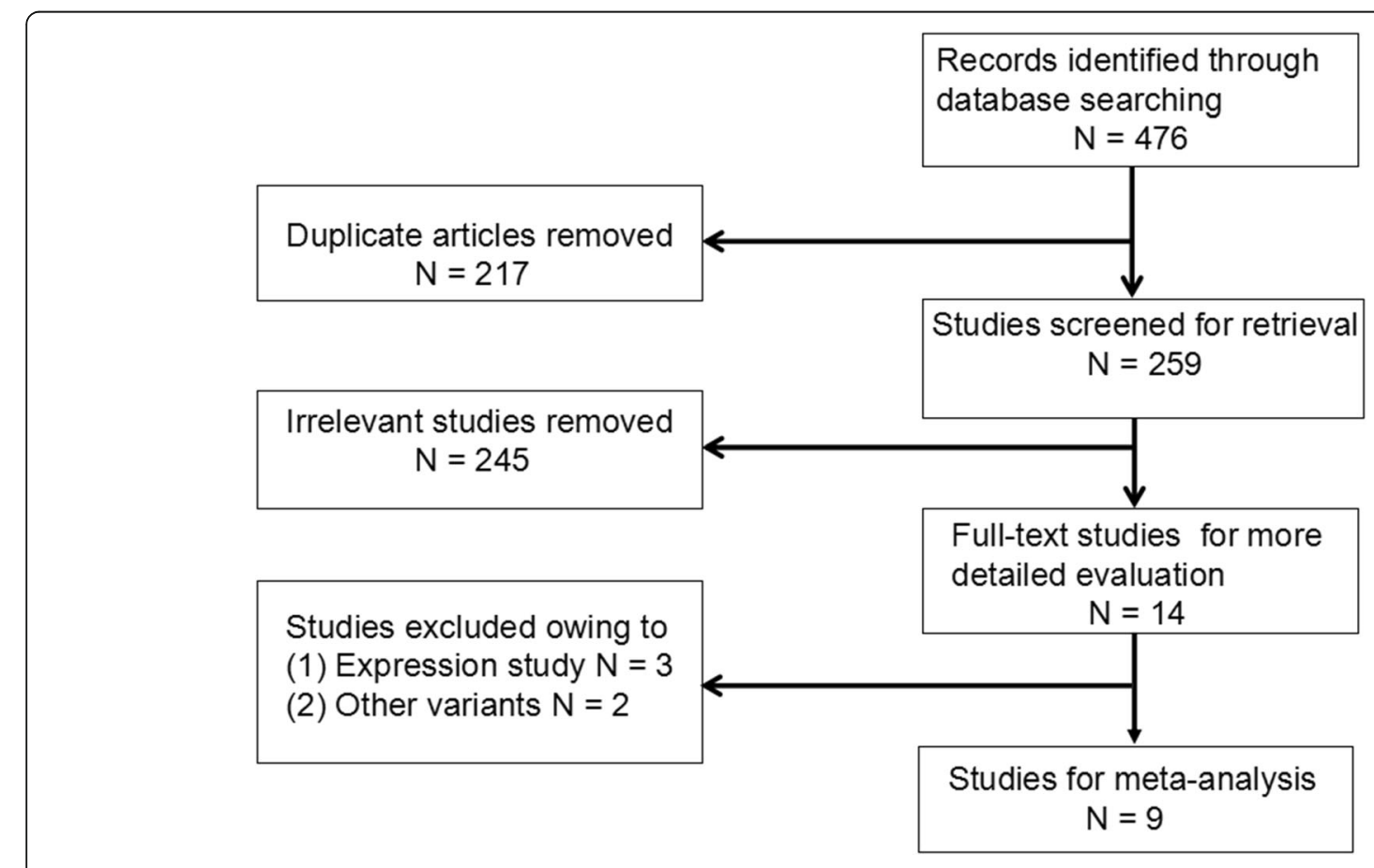

Fig. 1 Flow diagram of studies considered for inclusion 


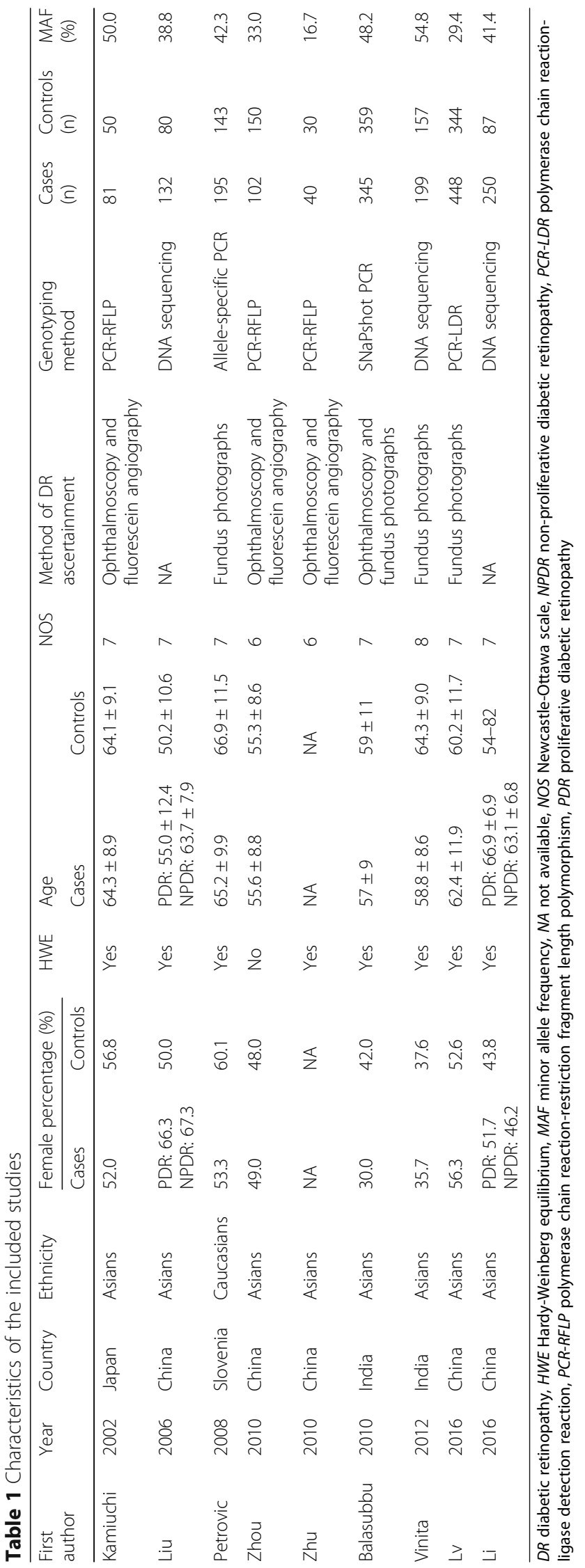


Table 2 Meta-analysis of the association between rs5498 and DR in type 2 diabetes

\begin{tabular}{|c|c|c|c|c|c|}
\hline Evaluation & Number of studies & OR $(95 \% \mathrm{Cl})$ & $P$ & P for heterogeneity & $1^{2}(\%)$ \\
\hline \multicolumn{6}{|c|}{$G A+G G$ versus $A A$} \\
\hline Total & 9 & $1.00(0.66-1.50)$ & 0.987 & $<0.001$ & 84.0 \\
\hline Asians & 8 & $0.96(0.61-1.50)$ & 0.843 & $<0.001$ & 85.2 \\
\hline Caucasians & 1 & $1.40(0.86-2.27)$ & 0.173 & NA & NA \\
\hline PDR & 4 & $1.22(0.61-2.47)$ & 0.577 & $<0.001$ & 86.8 \\
\hline NPDR & 3 & $0.60(0.29-1.21)$ & 0.151 & 0.007 & 80.0 \\
\hline \multicolumn{6}{|c|}{$\mathrm{GG}$ versus $\mathrm{GA}+\mathrm{AA}$} \\
\hline Total & 9 & $1.24(0.86-1.77)$ & 0.245 & 0.003 & 65.3 \\
\hline Asians & 8 & $1.15(0.79-1.68)$ & 0.469 & 0.008 & 63.2 \\
\hline Caucasians & 1 & $2.00(1.15-3.48)$ & 0.014 & NA & NA \\
\hline PDR & 4 & $1.90(0.80-4.50)$ & 0.146 & $<0.001$ & 84.2 \\
\hline NPDR & 3 & $0.92(0.58-1.47)$ & 0.724 & 0.477 & 0.0 \\
\hline \multicolumn{6}{|c|}{ GG versus $A A$} \\
\hline Total & 9 & $1.14(0.68-1.92)$ & 0.611 & $<0.001$ & 78.5 \\
\hline Asians & 8 & $1.04(0.60-1.81)$ & 0.887 & $<0.001$ & 78.1 \\
\hline Caucasians & 1 & $2.21(1.16-4.22)$ & 0.016 & NA & NA \\
\hline PDR & 4 & $1.91(0.64-5.73)$ & 0.246 & $<0.001$ & 87.6 \\
\hline NPDR & 3 & $0.77(0.47-1.25)$ & 0.286 & 0.171 & 43.5 \\
\hline \multicolumn{6}{|c|}{ G allele versus $A$ allele } \\
\hline Total & 9 & $1.08(0.81-1.45)$ & 0.592 & $<0.001$ & 85.7 \\
\hline Asians & 8 & $1.05(0.76-1.44)$ & 0.790 & $<0.001$ & 86.3 \\
\hline Caucasians & 1 & $1.44(1.06-1.95)$ & 0.021 & NA & NA \\
\hline PDR & 4 & $1.34(0.71-2.52)$ & 0.364 & $<0.001$ & 82.5 \\
\hline NPDR & 3 & $0.71(0.43-1.17)$ & 0.180 & 0.011 & 77.9 \\
\hline
\end{tabular}

$C l$ confidence interval, $D R$ diabetic retinopathy, NA not applicable, NPDR non-proliferative diabetic retinopathy, $O R$ odds ratio, $P D R$ proliferative diabetic retinopathy

the gene encoding ICAM-1 is thought to be involved in the development of DR.

The current mete-analytic review was conducted to verify the genetic contribution of a common SNP, rs5498 in the $I C A M-1$ gene to retinopathy risk in type 2 diabetes. Our results showed a lack of association between the ICAM-1 rs5498 polymorphism and risk of retinopathy in type 2 diabetes. Subgroup analysis by ethnicity did not reveal any significant association in Asian populations. In addition, when DR were subdivided into two main stages: non-proliferative and proliferative, we found no association of the ICAM-1 rs5498 polymorphism with the risk of non-proliferative and proliferative DR.

The ICAM-1 rs5498 polymorphism results in substitution of an A with a $\mathrm{G}$ nucleotide and replaces lysine (K) with a glutamic acid (E). It is thought that the SNP affects mRNA splicing patterns that modify cell-cell interactions and influence inflammatory response [8]. Kamiuchi et al. initially reported a positive association between rs5498 genotypes and retinopathy in type 2 diabetes, using a very small sample size (81 cases and 50 controls) [12]. However, their findings were not replicated by all other studies on the topic. It was noteworthy that the study by Balasubbu et al. with 704 participants and the study by Lv et al. involving 782 participants were the two having relatively large sample sizes among the included studies, but they did not identify any statistically significant association of rs5498 with DR [17, 19]. We could not exclude the possibility that false-positive findings may be obtained from studies with small sample sizes like one conducted by Kamiuchi et al.

Combining published data from nine studies involving 3192 participants, this is the largest meta-analysis on the relationship between the ICAM-1 rs5498 polymorphism and retinopathy in type 2 diabetes. Previous meta-analyses on the same topic included fewer studies (Su et al., 2013, $n=5$; Sun et al., 2014, $n=7$; Fan et al., 2015, $n=7$ ) [21-23]. Results from this meta-analysis differed from those of the meta-analysis by $\mathrm{Su}$ et al., which found an association between rs5498 and DR in type 2 diabetes. This was probably because in addition to the five studies included by $\mathrm{Su}$ et al. [21], we included four recently published case-control studies and conducted the pooled analyses with a larger sample size $[22,23]$. Sun et al. and Fan et al. found no association of 


\begin{tabular}{|l} 
Study \\
$\mathrm{ID}$
\end{tabular}

Fig. 2 Forest plot for included studies evaluating the association between the ICAM-1 rs5498 polymorphism and diabetic retinopathy in type 2 diabetic patients under a dominant model ( $G G+A G$ versus $A A)$. OR, oadds ratio; Cl, confidence interval

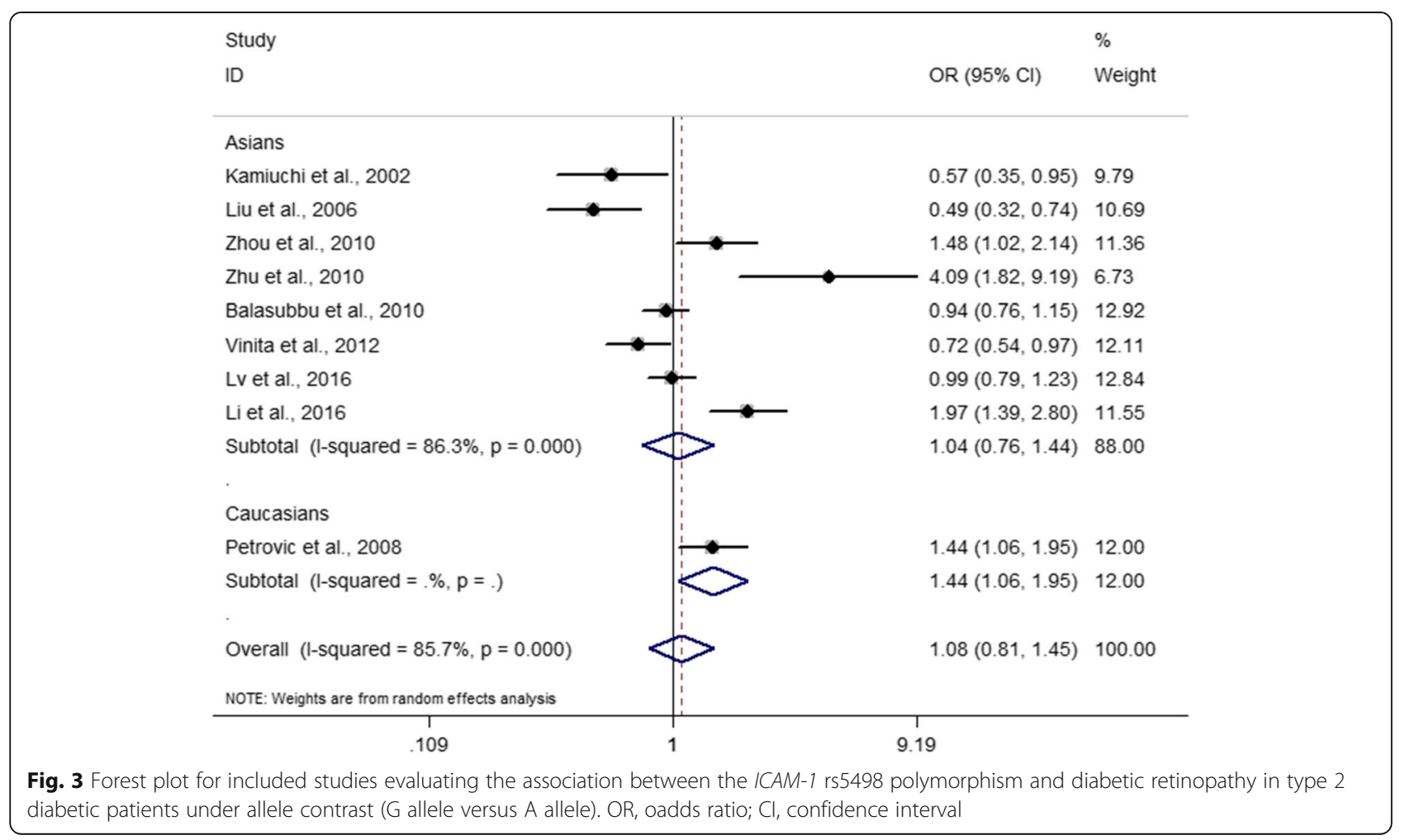




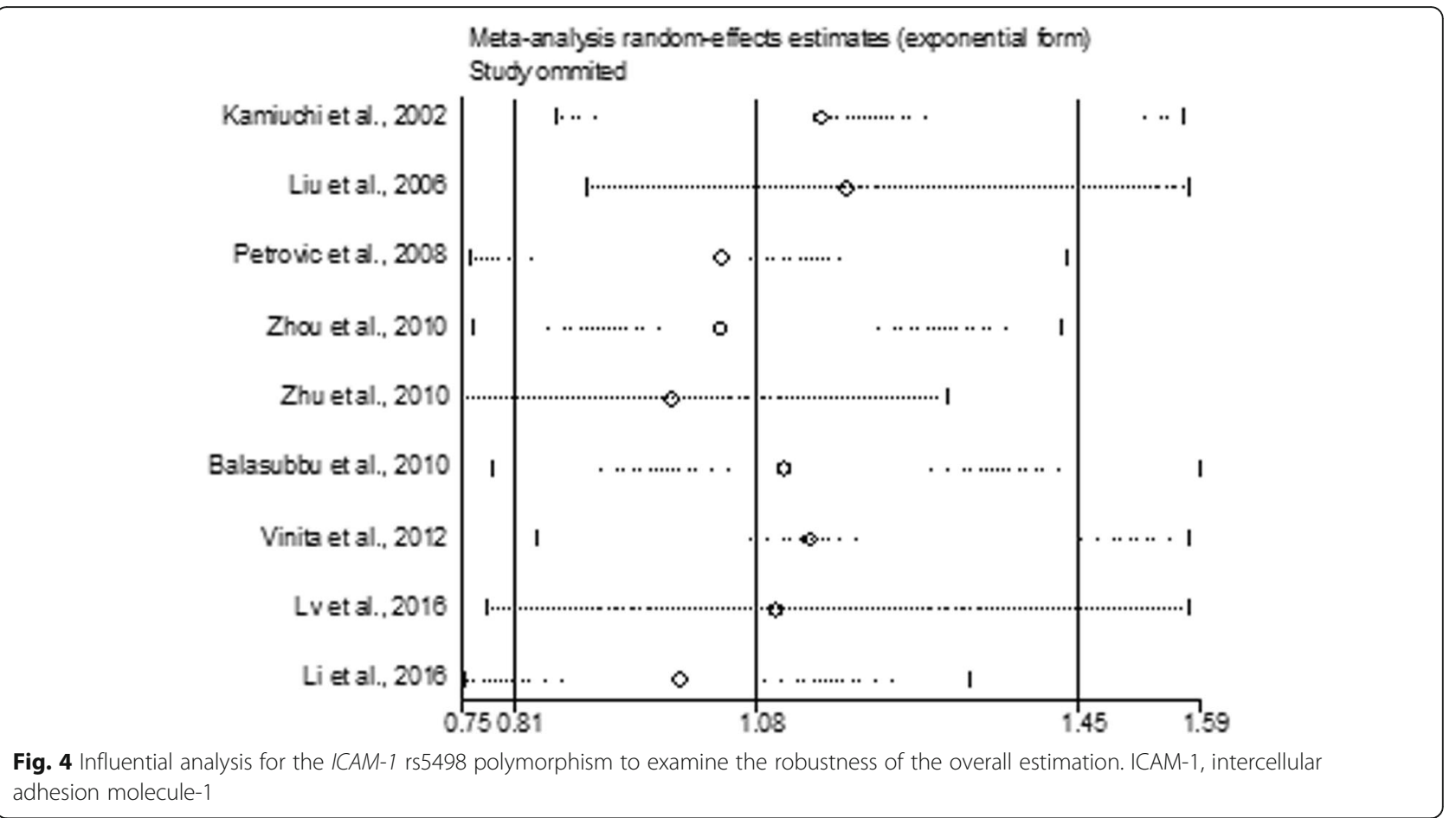

rs5498 with DR; their results were consistent with our calculations. Compared to the previous meta-analyses, our study had several strengths. First, considering that the cause and development of type 1 diabetes and type 2 diabetes were different, we only included retinopathy subjects of type 2 diabetes as cases in our analyses. We did not take into account the results from type 1 diabetes. Second, in addition to subgroup analyses by ethnicity, we performed subtype-specific analyses to evaluate the relation of rs5498 with the risk of non-proliferative and proliferative DR, respectively. Such evaluations were not performed by the previous meta-analyses. Third, we performed influential analysis to ensure the robustness of our combined estimations.

Since most of the included studies were conducted on Asian populations $(n=8)$, it became evident from this meta-analysis that further studies should include larger

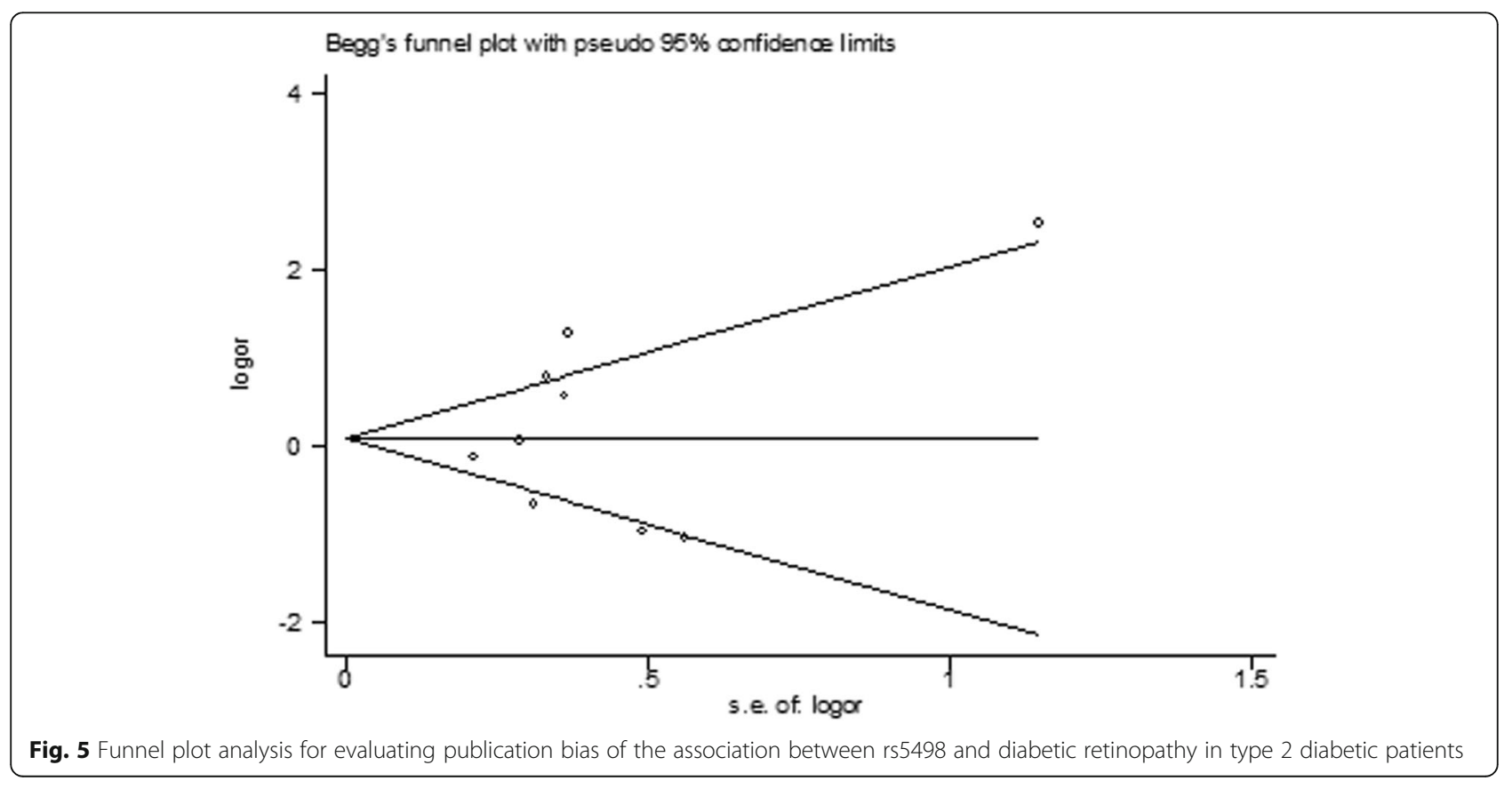


Table 3 Assessment of publication bias using Egger's test

\begin{tabular}{lllll}
\hline & $\begin{array}{l}\text { Dominant } \\
\text { model }\end{array}$ & $\begin{array}{l}\text { Recessive } \\
\text { model }\end{array}$ & $\begin{array}{l}\text { GG versus AA } \\
\text { contrast }\end{array}$ & $\begin{array}{l}\text { G allele versus A } \\
\text { allele contrast }\end{array}$ \\
\hline$P$ value & 0.754 & 0.498 & 0.641 & 0.579 \\
\hline
\end{tabular}

non-Asian populations to evaluate race-specific effects of rs5498, such as African and Caucasian populations. The study by Petrovic et al. was the only one performed in Caucasians [14]; their findings should be replicated in other Caucasian populations, including British, German, and French populations. Besides rs5498, more than 200 polymorphisms have been identified in the ICAM-1 gene. We could not exclude the possibility that other ICAM-1 polymorphisms played a role in the susceptibility to type 2 DR. The study by Simões et al. identified a significant association between the ICAM-1 rs1801714 polymorphsim and non-proliferative DR in type 2 diabetes [24]. In addition, a Chinese study assessed the association of DR with the rs1799969 polymorphism which was located in exon 4 of the ICAM-1 gene [25]. Because the evidence base for other ICAM-1 polymorphisms was very small at the time of planning our meta-analysis and consequently we chose to focus our analysis only on the rs 5498 polymorphism. It would be advisable that in the future attention should be paid to the relationship between other ICAM-1 polymorphisms and DR in type 2 diabetes.

Several limitations should be considered. First, this study was limited by the unavailability of individual patient data that would allow the identification of potential interactions of rs5498 with specific disease characteristics, including glycemic control, blood pressure control, and hyperlipemia. These factors might have contributed to the lack of success in identifying positive results in association studies for rs5498. Second, like all meta-analyses, the present study was susceptible to reporting biases. Despite the use of comprehensive strategies to identify eligible studies for rs 5498 , we could not exclude the possibility that some studies might have been erroneously excluded. Third, we did not evaluate the relation of serum soluble ICAM-1 (sICAM-1) levels with DR because of limited published data. There was some evidence suggesting that circulating sICAM levels were positively associated with DR prevalence in type 2 diabetes $[14,26]$. It is recommended that future association studies should perform sICAM-1 measurement and evaluate the effects of rs5498 on sICAM levels in DR patients, which could help clarify the role of rs5498 in DR development.

In conclusion, Our meta-analysis has shown that there is no significant association of the ICAM-1 rs5498 polymorphism with DR in type 2 diabetes. Further investigation involving non-Asian populations is warranted on the association between this polymorphism and DR, particularly studies with larger sample size that adjust for confounding variables.

\section{Additional files}

Additional file 1: Search strategy used in Pubmed. (DOC $27 \mathrm{~kb}$ )

Additional file 2: PRISMA Checklist. (DOC 57 kb)

\section{Abbreviations}

BMI: Body mass index; Cl: Confidence interval; DR: Diabetic retinopathy; HWE: Hardy-Weinberg equilibrium; ICAM-1: Intercellular adhesion molecule-1; MAF: Minor allele frequency; OR: Odds ratio; SNP: Single-nucleotide polymorphism

\section{Acknowledgements}

Not applicable.

\section{Funding}

This research receives no funding.

\section{Availability of data and materials}

All the data supporting our findings is contained within the manuscript.

\section{Author's contributions}

Conceived and designed the study: ZX and HL; Data acquisition: ZX; Analysis and interpretation: ZX and HL; Drafting the manuscript: ZX; Revising the manuscript critically: ZX and HL. Both authors made substantial contribution to this manuscript meeting authorship criteria, agreed to be accountable for all aspects of the work and have read and approved the final version.

Ethics approval and consent to participate Not applicable.

\section{Consent for publication}

Not applicable.

\section{Competing interests}

The authors declare that they have no competing interests.

\section{Publisher's Note}

Springer Nature remains neutral with regard to jurisdictional claims in published maps and institutional affiliations.

Received: 28 January 2018 Accepted: 29 October 2018

Published online: 12 November 2018

References

1. Eshaq RS, Aldalati AMZ, Alexander JS, Harris NR. Diabetic retinopathy: breaking the barrier. Pathophysiology. 2017;24:229-41.

2. Giloyan A, Harutyunyan T, Petrosyan V. The prevalence of and major risk factors associated with diabetic retinopathy in Gegharkunik province of Armenia: cross-sectional study. BMC Ophthalmol. 2015;15:46.

3. Gu HF, Ma J, Gu KT, Brismar K. Association of intercellular adhesion molecule 1 (ICAM1) with diabetes and diabetic nephropathy. Front Endocrinol (Lausanne). 2013:3:179.

4. Miyamoto K, Khosrof S, Bursell SE, Rohan R, Murata T, Clermont AC, et al. Prevention of leukostasis and vascular leakage in streptozotocin-induced diabetic retinopathy via intercellular adhesion molecule-1 inhibition. Proc Natl Acad Sci U S A. 1999;96:10836-41.

5. Barouch FC, Miyamoto K, Allport JR, Fujita K, Bursell SE, Aiello LP, et al. Integrin-mediated neutrophil adhesion and retinal leukostasis in diabetes. Invest Ophthalmol Vis Sci. 2000;41:1153-8.

6. Matsuoka M, Ogata N, Minamino K, Matsumura M. Leukostasis and pigment epithelium-derived factor in rat models of diabetic retinopathy. Mol Vis. 2007;13:1058-65.

7. Zhang XL, Wen L, Chen YJ, Zhu Y. Vascular endothelial growth factor upregulates the expression of intracellular adhesion molecule-1 in retinal endothelial cells via reactive oxygen species, but not nitric oxide. Chin Med J. 2009:122:338-43.

8. Joob B, Wiwanitkit V. ICAM-1 K469E polymorphism, increased risk of neurocysticercosis occurrence and immunopathological defect. Arq Neuropsiquiatr. 2017;75:681. 
9. Moher D, Liberati A, Tetzlaff J, Altman DG, PRISMA Group. Preferred reporting items for systematic reviews and meta-analyses: the PRISMA statement. Int J Surg. 2010;8:336-41.

10. Stang A. Critical evaluation of the Newcastle-Ottawa scale for the assessment of the quality of nonrandomized studies in meta-analyses. Eur J Epidemiol. 2010;25:603-5.

11. DerSimonian R, Laird N. Meta-analysis in clinical trials. Control Clin Trials. 1986:7:177-88.

12. Kamiuchi K, Hasegawa G, Obayashi H, Kitamura A, Ishii M, Yano M, et al. Intercellular adhesion molecule-1 (ICAM-1) polymorphism is associated with diabetic retinopathy in type 2 diabetes mellitus. Diabet Med. 2002;19:371-6.

13. Liu L, Yu Q, Wang H, Zhang SX, Huang C, Chen X. Association of intercellular adhesion molecule 1 polymorphisms with retinopathy in Chinese patients with type 2 diabetes. Diabet Med. 2006;23:643-8.

14. Petrovic MG, Osredkar J, Saraga-Babić M, Petrovic D. K469E polymorphism of the intracellular adhesion molecule 1 gene is associated with proliferative diabetic retinopathy in Caucasians with type 2 diabetes. Clin Exp Ophthalmol. 2008;36:468-72.

15. Zhou Y, Fu P, Fu X. Study on the gene polymorphism of intercellular adhesion molecule-1 in type 2 diabetes mellitus patients with retinopathy J Chin Pract Diagn Ther. 2010;10:29-31.

16. Zhu J, Gao S, Chi X, Zhang Y, Lai X, Zhong W. Association between the intercellular adhesion molecule-1 (ICAM-1) K469E polymorphism and diabetic retinopathy. J Fujian Med Univ. 2010;44:190-3.

17. Balasubbu S, Sundaresan P, Rajendran A, Ramasamy K, Govindarajan G, Perumalsamy N, et al. Association analysis of nine candidate gene polymorphisms in Indian patients with type 2 diabetic retinopathy. BMC Med Genet. 2010;11:158.

18. Vinita K, Sripriya S, Prathiba K, Vaitheeswaran K, Sathyabaarathi R, Rajesh M et al. ICAM-1 K469E polymorphism is a genetic determinant for the clinical risk factors of T2D subjects with retinopathy in Indians: a population-based case-control study. BMJ Open. 2012;2.

19. Lv Z, Li Y, Wu Y, Qu Y. Association of ICAM-1 and HMGA1 gene variants with retinopathy in type 2 diabetes mellitus among Chinese individuals. Curr Eye Res. 2016;41:1118-22.

20. Li L, Yi X, Gu Y, Qian Y, Zheng K. Association of genetic polymorphism of VEGF, ICAM-1 K469E, EPO and TCF7L2 genes with diabetic retinopathy of type 2 diabetes mellitus in Uyghur population in Xinjiang. J Xinjiang Med Univ. 2016;39:1268-71.

21. Su X, Chen X, Liu L, Chang X, Yu X, Sun K. Intracellular adhesion molecule-1 K469E gene polymorphism and risk of diabetic microvascular complications: a meta-analysis. PLoS One. 2013;8:e69940

22. Sun $H$, Cong $X$, Sun $R$, Wang C, Wang X, Liu Y. Association between the ICAM-1 K469E polymorphism and diabetic retinopathy in type 2 diabetes mellitus: a meta-analysis. Diabetes Res Clin Pract. 2014;104:e46-9.

23. Fan WY, Liu NP. Meta-analysis of association between K469E polymorphism of the ICAM-1 gene and retinopathy in type 2 diabetes. Int J Ophthalmol. 2015;8:603-7.

24. Simões MJ, Lobo C, Egas C, Nunes S, Carmona S, Costa MÂ, et al. Genetic variants in ICAM1, PPARGC1A and MTHFR are potentially associated with different phenotypes of diabetic retinopathy. Ophthalmologica. 2014;232: $156-62$.

25. Yang X, Deng Y, Gu H, Ren X, Li N, Lim A, et al. Candidate gene association study for diabetic retinopathy in Chinese patients with type 2 diabetes. Mol Vis. 2014:20:200-14.

26. van Hecke MV, Dekker JM, Nijpels G, Moll AC, Heine RJ, Bouter LM, et al. Inflammation and endothelial dysfunction are associated with retinopathy: the Hoorn study. Diabetologia. 2005;48:1300-6.

\section{Ready to submit your research? Choose BMC and benefit from:}

- fast, convenient online submission

- thorough peer review by experienced researchers in your field

- rapid publication on acceptance

- support for research data, including large and complex data types

- gold Open Access which fosters wider collaboration and increased citations

- maximum visibility for your research: over $100 \mathrm{M}$ website views per year

At BMC, research is always in progress.

Learn more biomedcentral.com/submissions 